\title{
Constrictive bronchiolitis obliterans in patient with Castelman's disease
}

\author{
E. Radzikowska, J. Pawlowski, M. Chabowski, R. Langfort
}

ABSTRACT: Constrictive bronchiolitis obliterans in patient with Castelman's disease. E. Radzikowska, J. Pawlowski, M. Chabowski, R. Langfort.

A 37-year-old woman with hialin- vascular type Castelman's disease $(C D)$ localised in the retroperitoneal region, incompletely resected, developed progressive dyspnoea. The chest radiograph taken 3 months before the operation was normal. The chest CT scan revealed diffused bronchiectases, hyperinflation and air trapping. Pulmonary function tests disclosed severe obstructive impairment with hyperinflation. The bronchoscopic examination of the bronchial tree was normal. Cultures of sputum, bronchial washing and blood were negative. No pemphigus antibodies were found.
Mycoplasmal, chlamydial and viral infections were excluded. Histological examination of specimens obtained by open lung biopsy revealed bronchiolar inflammation, submucosal bronchial fibrosis with obliteration of bronchiolar lumen. Constrictive bronchiolitis obliterans (CBO) was diagnosed. Despite slight clinical and spirometric improvements that were achieved due to corticosteroid therapy, one year later she died as a result of respiratory failure.

It is widely known that patients with $\mathrm{CD}$ develop CBO during the course of paraneoplastic pemphigus. However we present the case of $\mathrm{CBO}$ and $\mathrm{CD}$ but without any symptoms of this condition.

Monaldi Arch Chest Dis 2005; 63: 4, 226-229.

Keywords: Constrictive bronchiolitis obliterans, Castelman's disease, angiofollicular lymph node hyperplasia.

National Tuberculosis and Lung Diseases Research Institute, Warsaw, Poland.

Correspondence: Dr. E. Radzikowska, National Tuberculosis and Lung Diseases Research Institute, Warsaw; Plocka 29 st.; Poland; e-mail: e.radzikowska@igichp.edu.pl

\section{Introduction}

Angiofollicular lymph node hyperplasia or Castelman's disease (CD) is an uncommon clinicopathological entity that results in unregulated growth of lymphoid tissue. The disease is neither purely reactive, nor purely neoplasmatic in nature. Hialin-vascular, plasma cell and mixed variant are basic histological subtypes of this disease, but most frequently the hialin-vascular type is observed. CD clinically comprises an unicentric and multi-centric form. Thoracic localisation mainly occurs in the unicentric form of CD. Retroperitoneal localization was described in less than $20 \%$ of patients [1]. Autoantibodies secreted by Castleman's tumours react against epidermal proteins and might be an essential factor in the pathogenesis of bronchiolar fibrosis and constrictive bronchiolitis obliterans (CBO) [2, 3, 4, 5, 6, 7, 8].

$\mathrm{CBO}$ is an inflammatory and fibrotic process occurring predominantly in the wall and connective tissue of membranous and respiratory bronchioles leading to narrowing of their lumens [9]. CBO represents a pattern of the reaction, not the specific disease entity. The disease may develop due to numerous factors, such as exposure to toxic fumes (nitrogen oxides, sulphur dioxide, ammonia, chlorine, phosgene), drugs (penicillamine), autoimmune (collagen vascular disease, primary biliary cirrhosis, Stevens-Johnson syndrome, ulcerative colitis, psoriatic arthritis, pemphigus vulgaris), neoplastic disorders (lymphoma, chronic lymphocytic leukaemia, thymoma, retroperitoneal sarcomas, Waldenström's macroglobulinemia and carcinoid), in the organ receptions (bone marrow, lung and heart transplants) or due to infections (adenoviral, influenza, measles, RSV, mycolplasmal, chlamydial). Cytokines and growth factors (transforming growth factor beta, tumour necrosis factor alpha, platelet derived growth factor) can contribute to the development of inflammation and fibrosis in the bronchiolar wall. Autoantibody mediated injury one of the suggested causes of CBO, occurring during the course of autoimmunological diseases and deposits of IgG in the bronchial epithelium have sometimes been observed. The pathogenesis of CBO in the course of neoplasm is obscure but immunological disturbances are a probable source of this condition $[4,6,10,11,12]$.

$\mathrm{CBO}$ accompanying $\mathrm{CD}$ is observed in the course of paraneoplastic pemphigus (PP). According to our best knowledge there is only one published case in which the CBO accompanied CD without symptoms of pemphigus [7]. Therefore, the aim of this paper is to present the second case of $\mathrm{CBO}$ with $\mathrm{CD}$.

\section{Case report}

A 37 year-old woman, smoking up to 10 cigarettes per day for 14 years (non-smoking for the last 6 months), was admitted to our department on $20^{\text {th }}$ October, 2001, with the aim of diagnosing increasing dyspnoea. Since childhood she has suf- 
fered from recurrent infections of the urinary tract requiring antibiotic treatment. In March 2001 she had a viral infection of the upper respiratory tract with a fever exceeding $38^{\circ} \mathrm{C}$, and since then she was not feeling well. The dyspnoea was gradually increasing. She was admitted to the district hospital, where her chest X-ray was normal, but pulmonary function tests showed a decreased ventilation reserve with predominance of obturation: VC2.5L (77\% pred), $\mathrm{FEV}_{1}-0.9 \mathrm{~L}$ (34\% pred). Subsequently she experienced abdominal pains. The ultrasound and CT scan investigations of the abdomen revealed a retroperitoneal lesion located in the region of the left kidney. In August 2001 the tumour was not radically resected. The pathological examination of tumour samples and tissue from the surgical margin displayed the hialin-vascular type of the Castelman's disease (figure 1.) On further observation her status deteriorated to the point where even slight exertion induced dyspnoea. Therefore, the patient was referred to our hospital.

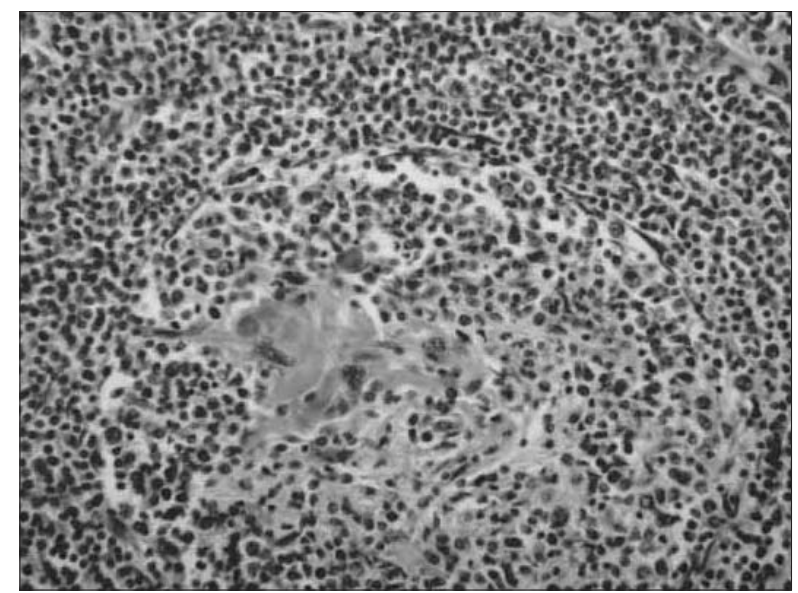

Fig. 1. - Follicular hyperplasia in the centre proliferation of hyalinised blood vessels that radiate through the germinal centres, and concentric array of small lymphocytes around the germinal centres (H\&E, x100).

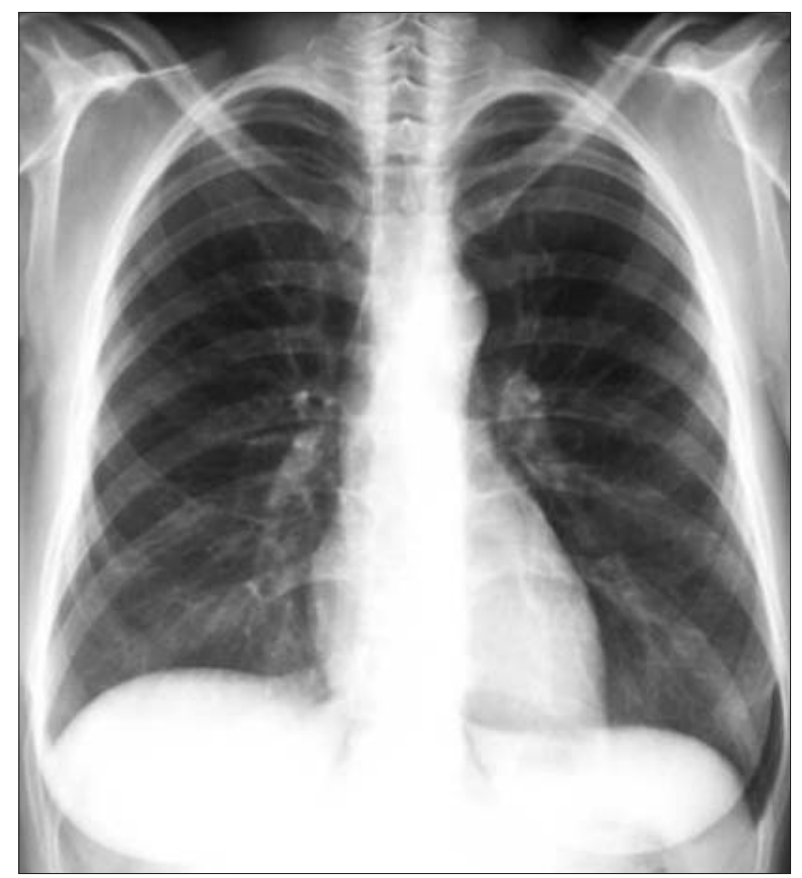

Fig. 2. - Chest radiograph nearly normal only slight peribronchial changes.
On admission she was fatigued and even movements in bed made her dyspnoeic. On physical examination the distal parts of her body were cyanotic. Tachycardia (110/min) and tachypnoea (22/min) were present. Auscultation revealed diminished breath sounds over both lungs. Numerous crepitant rales at the base of both lungs and many wheezes were heard during the examination. The chest X-ray film disclosed only slight peribronchial changes (figure 2). The HRCT chest scan indicated segmental and sub-segmental bronchiectases without mediastinal and hilar lymph node enlargement (figure 3). Expiratory HRCT showed air-trapping. Echocardiography demonstrated only slight tricuspid insufficiency. Pulmonary function tests displayed severe obturation without any reaction to vasodilative drugs, with evidence of pulmonary hyperinflation: FVC$1.79 \mathrm{~L}$ (55.6\% pred), $\mathrm{FEV}_{1}-0.52 \mathrm{~L}$ (18.7\% pred), $\mathrm{R}$ tot-1.5 $\mathrm{kPas} / \mathrm{L}(500 \%$ pred), ITGV-5.46 L (206\% pred), TLC-6.64 L (137\% pred); after inhalation of salbutamol: FVC-1.97 L (+9.8\%), $\mathrm{FEV}_{1}-0.52 \mathrm{~L}(+0 \%)$. An arterial blood gas sample (room air) showed arterial oxygen tension $\left(\mathrm{PaO}_{2}\right)$ of $8,4 \mathrm{kPa}(63.4 \mathrm{mmHg})$, arterial carbon dioxide tension $\left(\mathrm{PaCO}_{2}\right) 6,3 \mathrm{kPa}(47.1 \mathrm{mmHg}), \mathrm{HCO}^{-}-31$ $\mathrm{mmol} / \mathrm{L}, \mathrm{pH}$ 7.436. The CT scan of the abdomen revealed abnormal structures adjoining the iliopsoas muscle at a level of the lower part of the left kidney. It measured $2.5 \times 4.0 \mathrm{~cm}$ and was marginally intensified by adding contrast medium, suggesting a recurrence of a neoplasm. Serum tests for Mycoplasma pneumoniae, Chlamydia pneumoniae, Parainfluenza virus and adenovirus were negative. Also ANA, RF and pemphigus antibodies (to periplakin, evoplakin and bullous pemphigoid antigen) were negative as well. Diagnostic open lung biopsy was performed. Inflammatory and fibrous infiltrates were identified within bronchial and bronchiolar walls. The inflammatory infiltrates consisted mainly of lymphocytes and plasmocytes with oedema of a stroma and accumulation of foamy macrophages, which caused swelling of the bronchial wall, destruction of elastic fibres and considerable narrowing of the bronchial lumen. Within other bronchioles, the fibrosis and proliferation of muscular fibres was re-

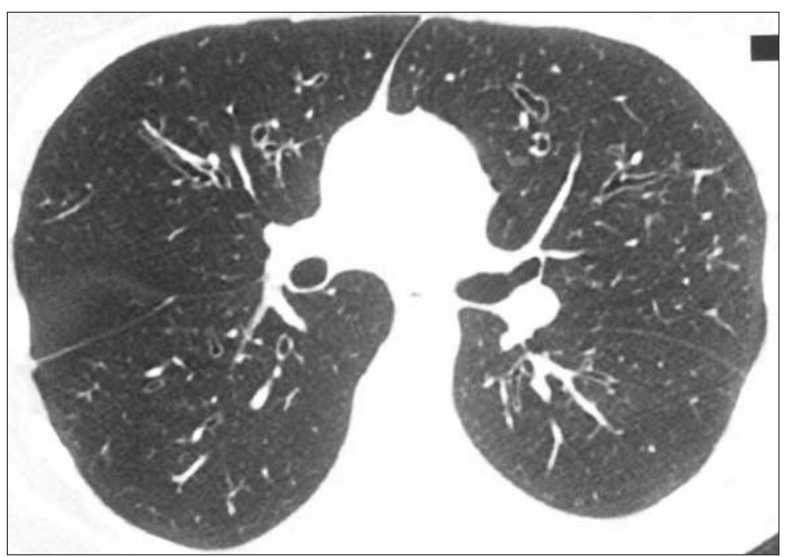

Fig. 3. - Inspiratory HRCT shows mild cylindrical bronchiectases in the upper lobes. 
vealed. The other bronchioles had total obstruction of their lumen. The alveolar structures were preserved (figure 4). The diagnosis of bronchiolitis obliterans was finally established. Immunohistochemical stains of pulmonary parenchyma for antigens of Herpes simplex virus, Cytomegalovirus, and Ebstein-Barr virus were negative. Methylprednisolone in a dose of $1 \mathrm{mg} / \mathrm{kg}$ body weight orally was introduced and a clinical improvement was observed. Control ultrasound examination of the abdomen determined a regression of the tumour in the location of the surgical procedure. After two months of treatment subsequent control spirometry showed a little improvement of ventilation parameters: FVC-1.8 L (55\% pred), $\mathrm{FEV}_{1}-0.55 \mathrm{~L}$ (19\% pred) and the dose of methylprednisolon was gradually reduced to $20 \mathrm{mg}$ daily. One year later she died in the course of exacerbation of the respiratory insufficiency in the district hospital.

\section{Discussion}

The presented case demonstrates the coexistence of two rare diseases, i.e. angiofollicular lymph node hyperplasia and CBO. In both cases the aetiology is supposed to be reactive $[1,10]$. In CD deregulation of the IL-6 output was shown. This interleukin controls the proliferation of Blymphocytes. Abnormal proliferation of B-lymphocytes and plasma cells causes overproduction of immunogolobulins, including autoantibodies [12]. Our patients had no elevated level of immunoglobulins and autoantibodies were not found either. The question of whether these diseases are induced by the same unidentified factor, or whether they are two independent diseases, is still open. So far, only one case of the isolated co-existence of $\mathrm{CD}$ and $\mathrm{CBO}$ has been presented in literature [7].

In other published cases $\mathrm{CBO}$ developed in the course of PP connected with CD. Nousari et al. observed respiratory failure with features of $\mathrm{CBO}$ in $30 \%$ of 84 cases with PP [8]. Similar coincidences of diseases were described by Canapllle et al., Wolff et al., Kim et al. and Chin et al. [2, 3, 4, 5]. The case of CBO concomitant with CD, PP and myasthenia gravis was observed by Chorzelski and co [6]. Autoantibodies secreted by Castleman's tumours, which react against epidermal proteins, could be an essential factor in the pathogenesis of PP. Paraneoplastic pemphigus autoantibodies against plakin proteins initiate the inflammatory process in the bronchiolar wall, and are responsible for bronchiolar fibrosis [6, 12]. Our patients had no symptoms of pemphigus and respective antibodies were not found.

Infections as a possible cause of CBO were excluded. No pathogens were cultured from lung specimens, bronchial washings and blood. The Herpes simplex virus and CMV were not detected by immunohistochemistry from the lung samples. Also, antibodies against Mycoplasma and Chlamydia pneumoniae were not found.

Moreover, according to clinical and serological findings the reported patient did not suffer from any

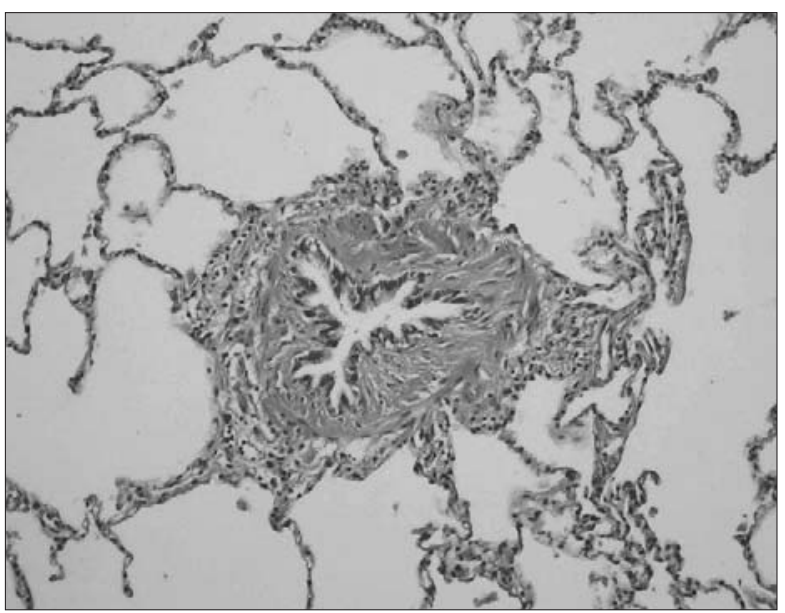

Fig. 4. - The lumen of the bronchiole is obliterated by fibrous connective tissue with infiltration of lymphoplasmocytes. The lung parenchyma is spared $(\mathrm{H} \& \mathrm{E}, \mathrm{x} 100)$.

collagen vascular disease, such as rheumatoid arthritis, systemic lupus erythematodes, sclerodermia.

Generally speaking, CBO is a disease of poor prognosis. All patients with $\mathrm{CBO}$ in the course of PP developed progressive respiratory failure and died despite aggressive immunosuppressive treatment [2, 3, 4, 6, 12]. Only Kondo et al. observed gradual recovery of respiratory symptoms after surgical removal of Castelman's tumour [7]. In our case excision of the tumour was incomplete. Steroid therapy slightly improved clinical status, ventilation and diminished the abdominal mass. This slight improvement was probably connected to the incomplete removal of the tumour and higher degree of bronchiolar fibrotic changes. She died of exacerbation of the respiratory failure one year later.

In our opinion, $\mathrm{CBO}$ developed during the course of $\mathrm{CD}$ in the presented case. Unidentified autoantibodies might be the probable cause of $\mathrm{CBO}$. However, it is possible that some type of infection, which was observed on the beginning of the illness, was the stimulus to the development of CBO.

In the future more detailed immunological assessment and other studies of cases with $\mathrm{CBO}$ and $\mathrm{CD}$ may elucidate a pathogenesis of $\mathrm{CBO}$ and will show new methods of effective treatment.

\section{References}

1. Barrie JR, English JC, Muller N. Castelman's disease of the lung: radiographic, high-resolution $\mathrm{CT}$ and pathologic findings. AJR 1996; 166: 1055-1056.

2. Caneppele S, Picart N, Bayle-Lebey P, et al. Paraneoplastic pemphigus associated with Castelman tumor. Clin Exp Dermatol 2000; 25: 219-21.

3. Wolff H, Kunte C, Messer G, et al. Paraneoplastic pemphigus with fatal pulmonary involvement in women with a mesenteric Castelman tumour. Br J Dermatol 1999; 140: 313-6.

4. Kim SC, Chang SN, Lee IJ, et al. Localized mucosal involvement and severe pulmonary involvenet in young patient with paraneoplastic pemphigus associat- 
ed with Castelman's tumor. Br J Dermatol 1998; 138: 667-71.

5. Chin AC, Stich D, White FV, Randhakrishnan J, Holterman MJ. Paraneoplastic pemphigus and bronchiolitis obliterans with mediastinal mass: A rare case of Castelman's disease with respiratory failure requiring lung transplantation. J Pediatr Surg 2001; 36: E12.

6. Chorzelski T, Hashimoto T, Maciejewska B, et al. Paraneoplastic pemphigus associated with Castelman tumor, myasthenia gravis and bronchiolitis obliterans. $J$ Am Acad Dermatol 1999; 41: 393-400.

7. Kondo M, Matsuda N, Chiyotani A, et al. A case o bronchobronchiolitis obilterans with Castelman's lymphoma. Nihon Kyobu Shikkan Gakkai Zaashi 1989; 27: 735-41.

8. Nousari HC, Deterding R, Wojtczak H, et al. The mechanism of respiratory failure in paraneoplastic pemphigus. New Engl J Med 1999; 340: 1406-1410.

9. Saito K, Morita M, Enomoto K. Bronchiolitis obliterans with pemphigus vulgaris and Cstelman's disease of hyaline vascular type: an autopsy case analyzed by computer aided 3-D reconstruction of airway lesions. Hum Pathol 1997; 28: 1310-1312.

10. Schlesinger C, Veeraraghavan S, Koss MN. Constrictive (obliterative) bronchiolitis. Curr Opin Pulm Med 1998; 4: 288-293.

11. Markopoulou KD, Cool CD, Elliot TL, et al. Obliterative bronchiolitis: varying presentations and clinicopathological correlation. Eur Respir J 2002; 19: 20-30.

12. Wang L, Bu D, Yang Y, Chen X, Zhu X. Castleman's tumours and production of autoantibody in paraneoplastic pemphigus. Lancet 2004; 363: 525-31.

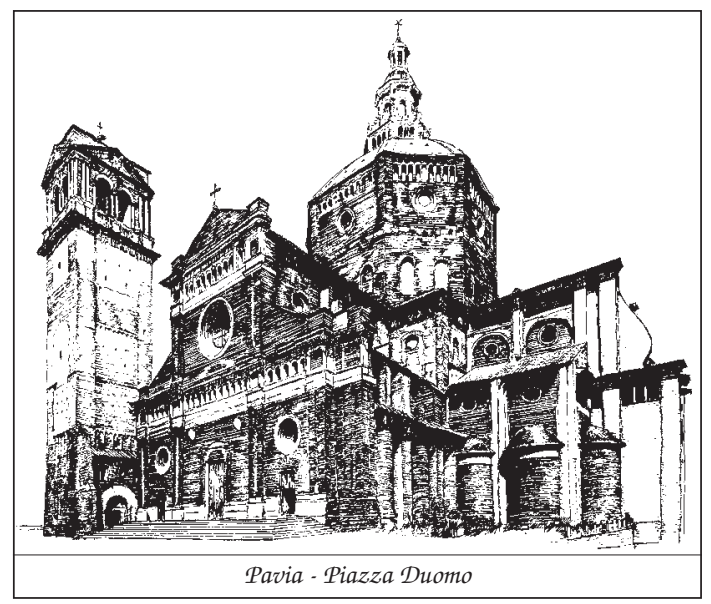

